\title{
Requirement of longer term antiviral therapy in patients with cytomegalovirus anterior uveitis with corneal endothelial cell damage
}

This article was published in the following Dove Press journal:

Clinical Ophthalmology

\author{
Yosuke Harada ${ }^{1,2}$ \\ Ken Fukuda' \\ Asami Nakahira' \\ Kentaro Tada' \\ Tamaki Sumi' \\ Atsuki Fukushima' \\ 'Department of Ophthalmology and \\ Visual Science, Kochi Medical School, \\ Nankoku, Japan; ${ }^{2}$ Department of \\ Ophthalmology and Visual Science, \\ Graduate School of Biomedical \\ Sciences, Hiroshima University, \\ Hiroshima, Japan
}

Background: The aim of the study was to investigate the efficacy of therapy in patients with cytomegalovirus (CMV) anterior uveitis.

Patients and methods: We reviewed the records of patients with CMV anterior uveitis who attended our institution between October 2010 and December 2015 and who were confirmed to have CMV DNA in the aqueous humor by polymerase chain reaction analysis.

Results: Fourteen immunocompetent patients (10 men and 4 women, total of 17 eyes) were enrolled. The mean $\pm \mathrm{SD}$ age at the onset of antiviral therapy was $63.1 \pm 11.3$ years (range, 44-87 years). CMV DNA was not detected in the aqueous humor of 3 patients on initial testing, but it was detected on subsequent analysis. All patients underwent systemic antiviral therapy. Among the patients who were followed up for more than 6 months after starting systemic antiviral therapy, systemic antiviral therapy was successfully terminated in all 4 patients without corneal endothelial loss but had to be continued because of disease recurrence on its termination in 5 of 8 patients $(62.5 \%)$ with corneal endothelial damage $(P=0.038)$.

Conclusions: Patients with corneal endothelial cell loss are likely to require longer term antiviral therapy than those without endothelial damage. In addition, whereas definitive diagnosis of CMV anterior uveitis requires the detection of CMV DNA in aqueous humor by polymerase chain reaction, one-fifth of patients in the present study tested negative on initial examination.

Keywords: cytomegalovirus, anterior uveitis, corneal endothelial cell, polymerase chain reaction, clinical course

\section{Introduction}

Cytomegalovirus (CMV) infection of the eye is well known to give rise to CMV retinitis in immunocompromised patients. Recently, however, such infection has also been found to give rise to CMV anterior uveitis in immunocompetent individuals. ${ }^{1-5} \mathrm{CMV}$ anterior uveitis is characterized by iridocyclitis with coin-shaped keratic precipitates, corneal endothelial cell loss, and elevation of intraocular pressure (IOP) (Figure 1). ${ }^{6}$ Definitive diagnosis of CMV anterior uveitis requires confirmation of the presence of CMV DNA in aqueous humor by polymerase chain reaction (PCR) analysis.

CMV anterior uveitis is treated with antiviral medications, such as oral valganciclovir or intravenous, intravitreal, or topical ganciclovir, together with topical steroids, ${ }^{7-9}$ although there is currently no consensus regarding the optimal treatment regimen. Given that intravitreal and topical antiviral therapy are off-label, oral valganciclovir is the first choice for treatment of this disease in Japan. ${ }^{7}$ Some studies have suggested that only topical antiviral therapy is able to control the condition. ${ }^{8,9}$ We investigated the clinical features of patients with CMV anterior uveitis attending $\mathrm{BY}$
hC for commercial use of this work, please see paragraphs 4.2 and 5 of our Terms (https://www.dovepress.com/terms.php). 

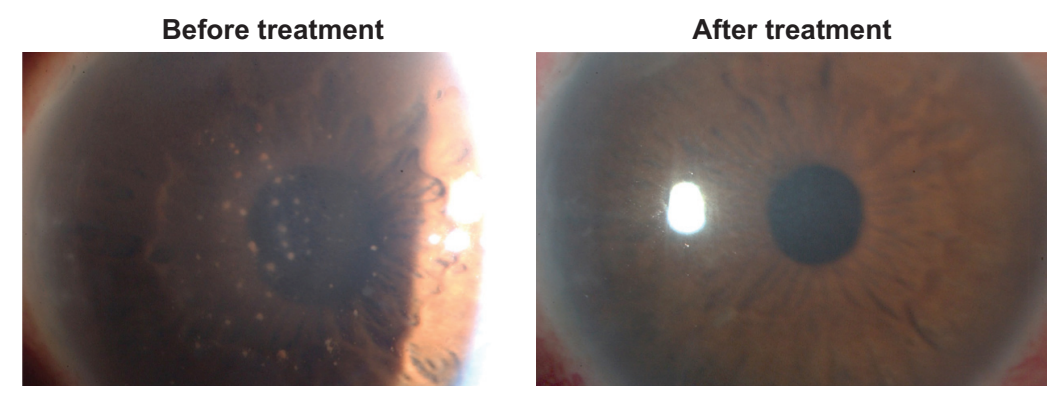

Figure I Slit-lamp microscopic photograph with cytomegalovirus anterior uveitis in Patient I showing numerous mutton fat keratic precipitations on the central to inferior corneal endothelium with mild stromal edema and elevation of intraocular pressure.

Notes: The anterior chamber contained 2+ cells according to the Standardization of Uveitis Nomenclature grading system. After antiviral treatment, these keratic precipitations and anterior chamber cells were resolved. Additionally, corneal transparency was restored.

our institution and evaluated the factors associated with poor prognosis.

\section{Patients and methods}

In this retrospective study, we reviewed patients with CMV anterior uveitis who were seen at Kochi Medical School between October 2010 and December 2015. The study adhered to the tenets of the Declaration of Helsinki and was approved by the Institutional Review Board of Kochi Medical School. It was granted a waiver of informed consent by the Institutional Review Board of Kochi Medical School because the data analyzed were de-identified records. The inclusion criteria were a diagnosis of CMV anterior uveitis confirmed by the detection of CMV DNA in aqueous humor by PCR analysis and negative findings for herpes simplex virus and varicella-zoster virus DNA. A $0.1 \mathrm{~mL}$ specimen of aqueous humor was aspirated with a 30-G needle and processed for determination of the copy number of CMV genomic DNA by real-time PCR analysis performed at SRL (Tokyo, Japan). Antiviral therapy was not administered before CMV DNA was detected by the PCR assay. If CMV DNA was detected in 1 eye and both eyes showed the same clinical features (anterior uveitis with coin-shaped lesions or corneal endothelial cell loss), the patient was diagnosed with bilateral disease. Patients who had already undergone corneal transplantation at the time of the confirmed diagnosis were excluded. Data collected included age, sex, date of diagnosis, medication history, eye examination findings, and follow-up period after starting antiviral medication. Many of the patients had previously attended other institutions, and records from these institutions were reviewed whenever possible.

CMV anterior uveitis was defined as active if the anterior chamber cell grade was $>1+$ according to the Standardization of Uveitis Nomenclature (SUN) grading system or if active granulomatous keratic precipitations were present. ${ }^{10}$
If the uveitis was diagnosed as active disease and PCR revealed positive CMV DNA, antiviral treatment was started. Our standard treatment for CMV anterior uveitis was oral valganciclovir (900 mg twice a day for 21 days and then tapered to $450 \mathrm{mg}$ twice a day) as well as topical 1\% ganciclovir eye drops (6 times a day). Topical steroid eye drops such as $0.1 \%$ fluorometholone or $0.1 \%$ betamethasone were used as needed. Given that long-term oral administration of valganciclovir can give rise to bone marrow suppression or impairment of fertility, we attempted to terminate such treatment if anterior uveitis was controlled with less than $0.5+$ inflammatory cells in the anterior chamber, as described by SUN working group grading system. ${ }^{10}$ For patients with contraindications such as renal failure or pancytopenia, either oral valganciclovir was administered at a lower dose or no oral antiviral treatment was given.

Corneal endothelial damage was defined as a corneal endothelial density (CED) of $<1,500$ cells $/ \mathrm{mm}^{2}$ regardless of ocular surgical history and was measured with the use of a specular microscope (NONCON ROB-C; Konan, Tokyo, Japan). Glaucoma was diagnosed in eyes with either glaucomatous optic disc changes or glaucomatous visual field defects.

Statistical analyses were performed using the JMP version 7.0.0 software program (SAS Institute, Cary, NC, USA). For statistical analysis with the chi-squared test, linear regression analysis with Spearman's correlation coefficient by rank test, and Mann-Whitney $U$ test, a $P$-value of $<0.05$ was considered statistically significant.

\section{Results}

Fourteen immunocompetent patients (10 men and 4 women, total of 17 eyes) were enrolled in the study (Table 1). Three patients $(18.8 \%)$ had bilateral involvement. Two patients had a history of other ocular diseases (sarcoidosis and scleritis, 


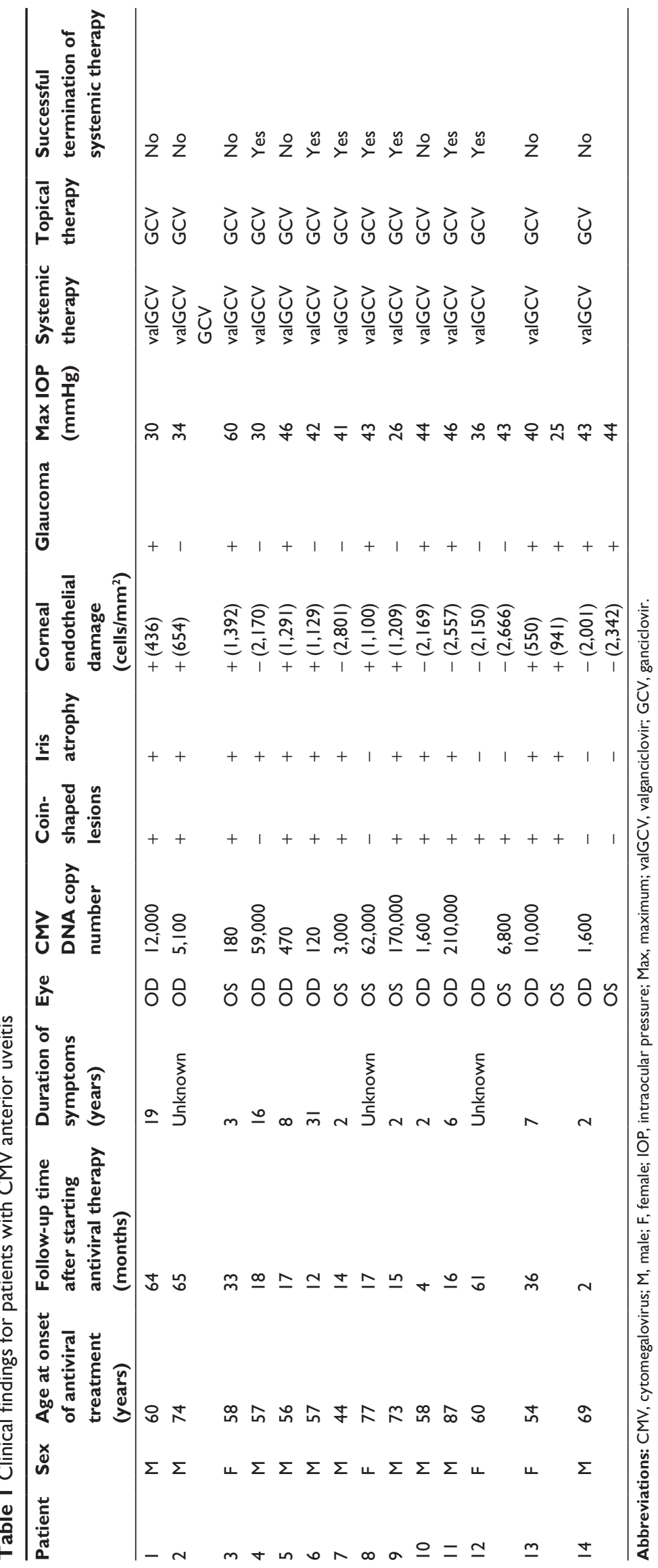


respectively). The mean age $\pm \mathrm{SD}$ at which patients started antiviral therapy was 63.1 \pm 11.3 years (range, 44-87 years), and the mean duration of follow-up by our institution was $36.7 \pm 22.9$ months (range, 4-67 months). The time of disease onset could be estimated for 11 of the 14 patients from the medical records, with the mean duration of disease for these individuals being $8.9 \pm 9.4$ years (range, 2-31 years). For the remaining 3 patients, the timing of disease onset could not be estimated according to the medical records from other institutions. Four patients (28.6\%) were diagnosed with PosnerSchlossman syndrome before attending our institution.

All patients were confirmed to have CMV DNA in the aqueous humor by PCR analysis. Three patients $(21.4 \%)$ tested negative for CMV DNA at the initial examination but tested positive at a subsequent examination. The copy number for CMV DNA did not correlate with either the time interval since the onset of disease or the CED $(P=0.6229$ and 0.9049 , respectively).

With regard to clinical features, 13 of the 17 eyes $(76.5 \%)$ manifested coin-shaped keratic precipitates, and 12 eyes (70.6\%) showed diffuse iris atrophy. The mean CED of these eyes was $1,661.9 \pm 775.0$ (range, $436-2,801$ ) cells $/ \mathrm{mm}^{2}$, and 9 eyes $(52.9 \%)$ exhibited a CED of $<1,500$ cells $/ \mathrm{mm}^{2}$. All eyes had ocular hypertension, with a mean maximum IOP of $39.7 \pm 8.7 \mathrm{mmHg}$ (range, $25-60 \mathrm{mmHg}$ ), and 10 eyes $(58.8 \%$ ) had glaucoma. Six of the 10 eyes with glaucoma underwent glaucoma surgery: trabeculectomy in 3 eyes, trabeculotomy in 2 eyes, and trabeculotomy followed by trabeculectomy in 1 eye. Four of these 6 eyes underwent glaucoma surgery before diagnosis of CMV infection. The presence of glaucoma surgical history also did not correlate with the CED $(P=0.5465)$.

All patients received oral valganciclovir as systemic antiCMV therapy. Only Patient 2 received intravenous ganciclovir followed by oral valganciclovir. All patients were also treated with $1 \%$ topical ganciclovir solution and topical corticosteroids. No patient was resistant to the antiviral therapy in this study. The ocular inflammation was resolved and the IOP decreased to a normal level after treatment onset in all 12 patients who were followed up for $>6$ months after treatment initiation (Patients 10 and 14 were excluded). However, 6 of these 12 patients $(50 \%)$ experienced disease recurrence during tapering or termination of anti-CMV therapy. Among 12 patients who were followed up for more than 6 months after starting systemic antiviral therapy (Patients 4, 5, 12, and 16 were excluded), systemic antiviral therapy was successfully terminated in 7 patients $(58.3 \%)$. The mean duration of using systemic antiviral therapy and the mean follow-up period after stopping systemic therapy in these patients

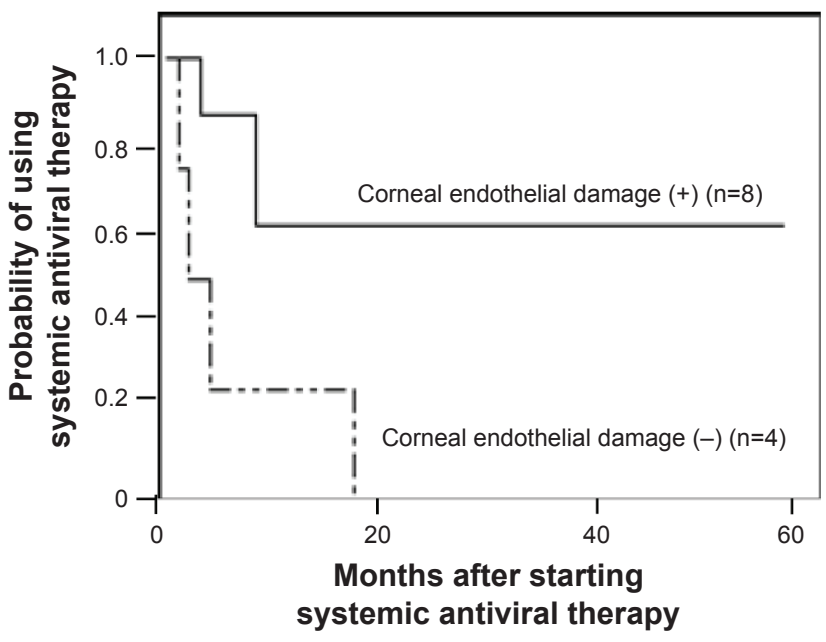

Figure 2 Kaplan-Meier analysis showing the probability of using systemic antiviral therapy in cytomegalovirus anterior uveitis.

Notes: In all 4 patients without corneal endothelial cell damage, systemic antiviral therapy could be terminated within 17 months, whereas 5 of the 8 patients $(62.5 \%)$ with corneal endothelial damage had to continue systemic therapy to prevent recurrent inflammation or intraocular pressure elevation $(P=0.023$, Wilcoxon signed-rank test).

were $6.1 \pm 5.5$ months (range, $1-17$ months) and 14.4 \pm 9.4 months (range, 7-33 months), respectively. Although all of these patients were being treated with topical ganciclovir solution to control inflammation, none of the 4 patients with out corneal endothelial cell loss needed to restart systemic antiviral therapy. However, 5 of the 8 patients $(62.5 \%)$ with corneal endothelial damage had to continue systemic therapy to prevent recurrent inflammation or IOP elevation ( $P=$ 0.038, chi-squared test) (Figure 2).

\section{Discussion}

CMV anterior uveitis is a newly identified form of uveitis characterized by recurrent anterior inflammation with corneal endotheliitis and IOP elevation. ${ }^{2,4}$ Although antiviral therapy for CMV is necessary for the treatment of this condition, no standard protocol has been established for such treatment, and few studies focusing on treatment outcome have been published. ${ }^{7,11}$ The findings of our study suggest that longer term systemic antiviral therapy is required to control ocular inflammation in patients with CMV anterior uveitis accompanied by corneal endothelial damage, than in patients without such damage, in order to prevent disease recurrence after treatment termination. Corneal endothelial damage may reflect the severity of inflammation and is thus a potential prognostic factor for CMV anterior uveitis.

The CMV viral load was previously found to be significantly associated with corneal endothelial cell loss, ${ }^{12}$ and the copy number of CMV DNA in aqueous humor was correlated with the severity of anterior segment inflammation ${ }^{13}$ 
in patients with CMV anterior uveitis. However, we did not detect a significant correlation between the CED and CMV DNA copy number in our patients. There are several possibilities to explain this discrepancy. First, this may have been due to the wide range of CMV DNA copy numbers (120-210,000) or to the small number of patients in our study. Second, we also outsourced the real-time PCR analysis of CMV genomic DNA to a commercial company, whereas such analyses were performed in the institution's own PCR lab in previous studies. ${ }^{12,13}$ The results may be influenced by the CMV DNA copy number of aqueous humor samples that were obtained at different time points, ie, aqueous humor was collected from some patients with active inflammation but from others with mild inflammation. Multiple samplings of aqueous humor from a single patient at different time points may be needed to more accurately determine the relationship between the CMV DNA copy number and CED. Finally, the discrepancy may have also been caused by the difference in the pathogenesis of CMV among the patients in this study. Some CMV strains may have strong affinity to the corneal endothelium, leading to high CED. A further study with a larger sample size, including genetic analysis of CMV causing anterior uveitis, is needed to address this possibility.

Definitive diagnosis of $\mathrm{CMV}$ anterior uveitis requires the detection of CMV DNA in aqueous humor by PCR. CMV DNA was eventually detected in all patients of our study, but about $20 \%$ of patients initially tested negative by PCR. Similar findings were reported in a previous study, ${ }^{14}$ indicating that repeat PCR testing should be considered if CMV anterior uveitis is clinically suspected despite an initial negative result for CMV DNA.

\section{Limitations}

There are several limitations of our study. First, the number of patients enrolled was small. To increase the number of eligible patients, we included individuals with CMV anterior uveitis regardless of their ocular surgical history or prior clinical complications such as scleritis or other forms of uveitis. A larger series of patients excluding such individuals will be needed to confirm that corneal endothelial cell loss is a risk factor for dependence on long-term systemic antiviral therapy. Analysis of a larger number of patients should also clarify the relation between CMV DNA copy number and disease severity. A second limitation of our study is that the patients were treated by several different ophthalmologists, some of whom were not uveitis specialists but instead had other specialties, such as the cornea or glaucoma, possibly resulting in some bias in treatment decisions.
Third, as already mentioned, real-time PCR analysis of CMV DNA was not performed in-house.

\section{Conclusion}

Our results suggest that patients with CMV anterior uveitis and corneal endothelial cell loss are likely to require longer term antiviral therapy than patients without endothelial damage. In addition, one-fifth of the patients with CMV anterior uveitis in our study tested negative for the presence of CMV DNA in aqueous humor at the initial analysis. Such PCR analysis should thus be repeated for patients with an initial negative result if CMV anterior uveitis is clinically suspected.

\section{Disclosure}

The authors report no conflicts of interest in this work.

\section{References}

1. Markomichelakis NN, Canakis C, Zafirakis P, Marakis T, Mallias I, Theodossiadis G. Cytomegalovirus as a cause of anterior uveitis with sectoral iris atrophy. Ophthalmology. 2002;109(5):879-882.

2. de Schryver I, Rozenberg F, Cassoux N, et al. Diagnosis and treatment of cytomegalovirus iridocyclitis without retinal necrosis. Br JOphthalmol. 2006;90(7):852-855.

3. Chee SP, Jap A. Presumed fuchs heterochromic iridocyclitis and PosnerSchlossman syndrome: comparison of cytomegalovirus-positive and negative eyes. Am J Ophthalmol. 2008;146(6):883-889.

4. Koizumi N, Yamasaki K, Kawasaki S, et al. Cytomegalovirus in aqueous humor from an eye with corneal endotheliitis. Am J Ophthalmol. 2006; 141(3):564-565

5. Chee SP, Bacsal K, Jap A, Se-Thoe SY, Cheng CL, Tan BH. Corneal endotheliitis associated with evidence of cytomegalovirus infection. Ophthalmology. 2007;114(4):798-803.

6. Chee SP, Bacsal K, Jap A, Se-Thoe SY, Cheng CL, Tan BH. Clinical features of cytomegalovirus anterior uveitis in immunocompetent patients. Am J Ophthalmol. 2008;145(5):834-840.

7. Koizumi N, Inatomi T, Suzuki T, et al; Japan Corneal Endotheliitis Study Group. Clinical features and management of cytomegalovirus corneal endotheliitis: analysis of 106 cases from the Japan corneal endotheliitis study. Br J Ophthalmol. 2015;99(1):54-58.

8. Wong JX, Agrawal R, Wong EP, Teoh SC. Efficacy and safety of topical ganciclovir in the management of cytomegalovirus (CMV)-related anterior uveitis. J Ophthalmic Inflamm Infect. 2016;6(1):10.

9. Koizumi N, Miyazaki D, Inoue T, et al. The effect of topical application of $0.15 \%$ ganciclovir gel on cytomegalovirus corneal endotheliitis. Br J Ophthalmol. 2017;101(2):114-119.

10. Jabs DA, Nussenblatt RB, Rosenbaum JT. Standardization of uveitis nomenclature for reporting clinical data. Results of the First International Workshop. Am J Ophthalmol. 2016;140(3):509-516.

11. Chee SP, Jap A. Cytomegalovirus anterior uveitis: outcome of treatment. Br J Ophthalmol. 2010;94(12):1648-1652.

12. Miyanaga M, Sugita S, Shimizu N, et al. A significant association of viral loads with corneal endothelial cell damage in cytomegalovirus anterior uveitis. Br J Ophthalmol. 2010;94(3):336-340.

13. Kandori M, Miyazaki D, Yakura K, et al. Relationship between the number of cytomegalovirus in anterior chamber and severity of anterior segment inflammation. Jpn J Ophthalmol. 2013;57(6):497-502.

14. Sobolewska B, Deuter C, Doycheva D, Zierhut M. Long-term oral therapy with valganciclovir in patients with Posner-Schlossman syndrome. Graefes Arch Clin Exp Ophthalmol. 2014;252(1):117-124. 


\section{Publish your work in this journal}

Clinical Ophthalmology is an international, peer-reviewed journal covering all subspecialties within ophthalmology. Key topics include: Optometry; Visual science; Pharmacology and drug therapy in eye diseases; Basic Sciences; Primary and Secondary eye care; Patient Safety and Quality of Care Improvements. This journal is indexed on

Submit your manuscript here: http://www.dovepress.com/clinical-ophthalmology-journal
PubMed Central and CAS, and is the official journal of The Society of Clinical Ophthalmology (SCO). The manuscript management system is completely online and includes a very quick and fair peer-review system, which is all easy to use. Visit http://www.dovepress.com/ testimonials.php to read real quotes from published authors. 